\title{
Effect of COVID-19 on Canadian Medical Student Attitudes toward Ophthalmology Residency Application
}

\author{
Cody Lo, $\mathrm{BSC}^{1}$ Amandeep Rai, MD ${ }^{2,3}$ Jonathan A. Micieli, MD, CM${ }^{2,3}$
}

${ }^{1}$ Faculty of Medicine, University of British Columbia, Vancouver, British Columbia, Canada

2 Department of Ophthalmology and Vision Sciences, University of

Address for correspondence Jonathan A. Micieli, MD, CM, Kensington Vision and Research Centre, Department of Ophthalmology and Vision Sciences at the University of Toronto, Suite 501340 College St. Toronto, Toronto, Ontario, Canada

${ }^{3}$ Kensington Vision and Research Centre, Department of Toronto, ON (e-mail: jmicieli@kensingtonhealth.org).

Ophthalmology and Vision Sciences at the University of Toronto,

Toronto, Ontario, Canada

J Acad Ophthalmol 2021;13:e5-e10.

\begin{abstract}
Keywords

- ophthalmology

- medical education

- residency

Objective This study aims to survey medical students interested in ophthalmology about how changes in electives and Canadian Residency Matching Service (CaRMS) due to the novel coronavirus disease 2019 (COVID-19) pandemic have affected their attitudes toward applying to ophthalmology residency.

Design This is a cross-sectional survey.

Participants A total of 32 Canadian medical students interested in ophthalmology responded to the survey.

Methods A 32-question survey tool used was developed in consultation with medical students, academic ophthalmologists, and residency program directors. The survey was distributed through e-mail by local ophthalmology interest groups at all of Canada's medical schools.

Results Respondents felt that changes in ability to travel for electives significantly decreased their likelihood of applying to ophthalmology residency. Additionally, respondents expressed concerns that lack of travel for electives and in-person CaRMS interviews significantly reduced their chances of successfully matching to ophthalmology. Respondents identified one-on-one video calls with program directors and residents as the initiatives that would best counteract the negative impacts from COVID-19. Increased presence of programs on social media were relatively less valued. Conclusion Canadian medical students interested in ophthalmology have concerns about how changes in electives and the CaRMS match due to COVID-19 will impact their ability to be fairly assessed and successfully match to ophthalmology. Lack of travel for electives and interviews has also resulted in students feeling ill equipped to make informed choices about program selection. However, there remains a sustained interest in ophthalmology among applicants.
\end{abstract}

received

October 16, 2020

accepted after revision

December 15, 2020
DOI https://doi.org/

10.1055/s-0040-1722740.

ISSN 2475-4757. (c) 2021. The Author(s).

This is an open access article published by Thieme under the terms of the Creative Commons Attribution-NonDerivative-NonCommercial-License, permitting copying and reproduction so long as the original work is given appropriate credit. Contents may not be used for commercial purposes, or adapted, remixed, transformed or built upon. (https://creativecommons.org/ licenses/by-nc-nd/4.0/)

Thieme Medical Publishers, Inc., 333 Seventh Avenue, 18th Floor, New York, NY 10001, USA 
The final year of medical school represents an important transition from undergraduate to postgraduate medical training. In Canada, medical students typically complete some combination of core and elective clinical rotations and participate in the R-1 match for postgraduate training programs through the Canadian Residency Matching Service (CaRMS). ${ }^{1}$ Canadian medical students would get opportunities to explore specialties and programs of interest through elective rotations where they would get clinical exposure working with a specific department. Students would also get exposure through the CaRMS interviews which were typically held in person at the host institution and included social events with current residents. The 2021 CaRMS cycle represents a dramatic shift in this process due to limitations as a result of the novel coronavirus disease 2019 (COVID-19) pandemic. $^{2,3}$ The COVID-19 has resulted in several changes to the 2021 CaRMS match as follows: (1) all interviews being held virtually, (2) no out-of-province electives, and (3) altered elective availability at a student's home institution.

For medical students interested in ophthalmology, these challenges are compounded by the fact that ophthalmology is consistently ranked as one of the most competitive specialities. ${ }^{4}$ There seems to be a significantly lower likelihood of matching to a competitive program in the first iteration of the CaRMS match when applying from a medical school in Western Canada or Quebec compared with Ontario. ${ }^{5}$ These discrepancies could potentially be exacerbated by limitations on travel between Canadian provinces. COVID-19 has also had a profound impact on many aspects of academic ophthalmology including didactic teaching, surgical volumes, research, and academic administration. ${ }^{6}$ The majority of research with respect to matching to an ophthalmology residency is performed on the SF Match (The San Francisco Match) that occurs in the United States which differs significantly from the CaRMS match. ${ }^{7-9}$ In contrast to the U.S. match, CaRMS does not place an emphasis on quantitative cognitive measures, such as scores on the USMLE (United States Medical Licensing Examination) step 1 which has been associated with successfully matching to ophthalmology. ${ }^{10}$

As programs and students navigate the new transient and potentially permanent changes to electives and the CaRMS match, there has been little empiric investigation of the perspectives of Canadian medical students interested in ophthalmology. This study aims to survey Canadian medical students about how changes in electives and travel limitations change their attitudes toward applying to ophthalmology and what they would like to see from programs.

\section{Methods}

\section{Sample Selection}

This was a cross-sectional survey of medical students interested in applying to ophthalmology residency at Canadian medical schools. An "interest" was defined here as a selfreported potential that the participant would apply to a Canadian ophthalmology residency program. Participants responded anonymously to the survey. Participants had to be over 18 years old and able to complete the survey in English. This study was approved by the University of Toronto Ethics Board (Protocol no.: 39654).

\section{Survey Development}

Despite published surveys of medical student perspectives on matching to residency in fields, such as neurosurgery, otolaryngology, and urology during the COVID-19 pandemic, there are no psychometrically validated questionnaires in this area. ${ }^{11-13}$ Given the novelty and time sensitive nature of this topic, this survey was developed following review of published surveys on medical student perspectives related to the COVID-19 pandemic both generally and specifically related to residency matching. ${ }^{11,14}$ Stakeholders involved in the creation of the research tool included experts in ophthalmology medical education, senior medical students, and residency program directors. The survey contained a mixed of quantitative and qualitative responses. All qualitative responses were optional but ensured our survey captured perspectives of medical students who were not addressed in the quantitative questions. The survey was created using Google Forms. Validity of responses were assessed by asking two factual questions about elective capacity to assess whether respondents were giving informed responses. Layout of the questionnaire was informed by the best practices for survey research. ${ }^{15}$ For example, the survey was separated into three main sections based on themes of questions asked (-Supplemental Material s1; online only). The final questions and survey tool were pilot tested and validated for usability and clarity by 10 senior medical students who did not partake in the actual survey study. The time required to complete the survey was approximately 5 to 10 minutes.

\section{Survey Distribution}

A purposive nonrandom sampling technique was used. Student interest groups in ophthalmology or surgery at all of Canada's 17 medical schools were contacted with a request to distribute our survey among their members across all years of their respective programs. Snowball sampling was also utilized as a mean to obtain a sufficiently large sample of medical students interested in ophthalmology. A reminder e-mail was sent after 2 weeks to maximize the response rate. The survey was distributed via an online link which had a landing page that clearly explained the context of the study. All responses were anonymous with no tracking of e-mail addresses or identifying information. Additionally, a cover letter was attached with a consent form that provided the contact information of the study team and other relevant details. The survey was open for 3 weeks and closed in August 2020.

\section{Data Analysis}

Mean participant response on the -3 to +3 scale was calculated for all questions where a score of 0 was considered "no change." Statistical differences were determined using the one sample $t$-test with a score of 0 as a hypothetical population mean. Quantitative data were analyzed using Microsoft Excel for Mac (Version 16.40, Redmond, WA) and 
Table 1 Participants demographics $(n=32)$

\begin{tabular}{|c|c|}
\hline Demographic & Number of respondents (\%) \\
\hline \multicolumn{2}{|l|}{ Medical school } \\
\hline - University of Alberta & $3(9)$ \\
\hline - University of British Columbia & $8(25)$ \\
\hline -Dalhousie University & $1(3)$ \\
\hline - University of Manitoba & $1(3)$ \\
\hline • McGill University & $1(3)$ \\
\hline - McMaster University & $4(13)$ \\
\hline - Memorial University & $1(3)$ \\
\hline -Queen's University & $3(9)$ \\
\hline - University of Toronto & $9(28)$ \\
\hline - Université de Montréal & $1(3)$ \\
\hline \multicolumn{2}{|l|}{ Gender } \\
\hline •Male & $21(66)$ \\
\hline •Female & $10(31)$ \\
\hline - Prefer not to say & $1(3)$ \\
\hline \multicolumn{2}{|c|}{ Number of specialties planning to apply to } \\
\hline$\cdot 1$ (ophthalmology only) & $12(38)$ \\
\hline$\cdot 2$ & $14(44)$ \\
\hline - 3 or more & $5(16)$ \\
\hline - Not sure & $1(3)$ \\
\hline
\end{tabular}

summarized using descriptive statistics. A p-value of $\leq 0.05$ was considered to be statistically significant. Qualitative data were analyzed by a single author using thematic analysis to identify recurrent ideas discussed by the cohort. Recurrent ideas were grouped into larger themes that described the perspective of medical students.

\section{Results}

A total of 32 medical students responded to the survey, representing a response rate of $43 \%$ based on previous 2020 CaRMS statistics. ${ }^{1}$ This response rate is considered adequate for surveys in which there is only a short time period available to collect data. ${ }^{16}$ Demographic information of respondents is summarized in - Table 1. The majority of participants (94\%) were senior medical students graduating in 2021 or 2022. A total of 10 Canadian medical schools were represented with distribution among schools roughly in keeping with relative class sizes. The majority of respondents were planning on either applying only to ophthalmology (38\%) or to ophthalmology and one other specialty (44\%) in the CaRMS match.

Responses to questions about how changes in electives and interviews due to COVID-19 have impacted medical student attitudes toward applying to ophthalmology residency are summarized in - Fig. 1. The ability of respondents to pursue electives at different programs within the same province ( $p=0.0039)$ and other provinces $(p=0.0071)$ had a significant negative impact on likelihood of applying to ophthalmology residency. Respondents also felt that lack of travel for electives ( $p=0.0065)$, in-person CaRMS interviews $(p=0.0034)$, and changes to medical education outside of COVID-19 $(p=0.0267)$ significantly reduced their chances of successfully matching to ophthalmology.

When asked what residency programs could do to counteract negative impacts from COVID-19, all initiatives and social media platforms were found to have a statistically significant benefit $(p<0.0001)$. Initiatives that respondents valued most highly were one-on-one calls with program directors (3.94 out of 5) and residents (3.97 out of 5). Other initiatives included invitation to department grand rounds (3.28 out of 5), online program information sessions (3.19 out of 5), and video tours of facilities (3.06 out of 5). Presence on social media was generally less valued compared with other initiatives with Facebook being rated highest ( 2.44 out of 5) followed by Instagram (2.34 out of 5), Twitter (2.19 out of 5), and Linkedin (1.91 out of 5).

Qualitative analysis revealed reassurance that changes would affect all medical students to a similar degree and that respondents' value equity in the CaRMS process regardless of the new restrictions:

"I would have personally preferred to have interviews in person although this has not effected (sic) my decisions since everyone will have to conduct their interviews through the same format."-Participant no. 28 

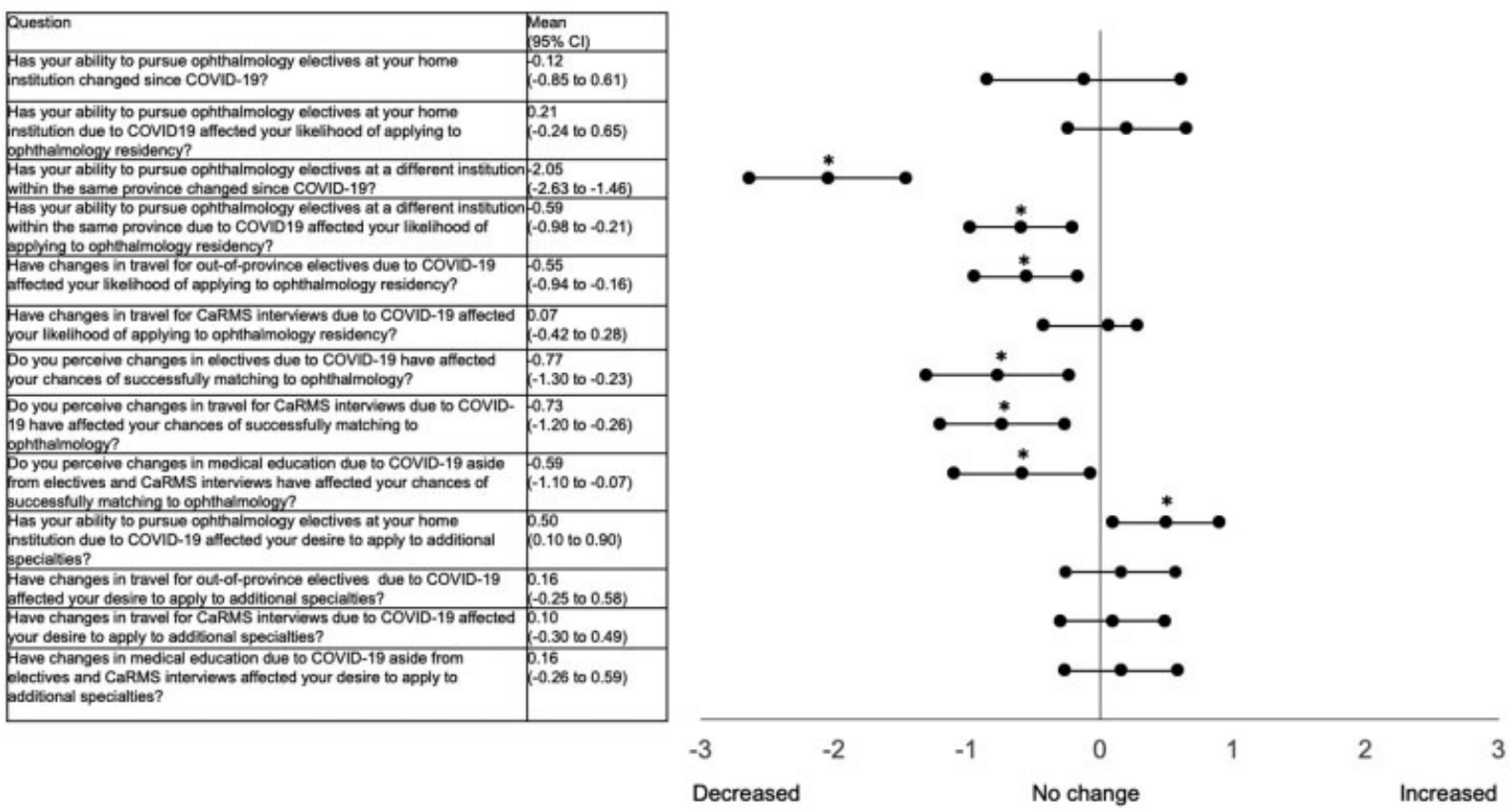

Fig. 1 The effect of COVID-19 on medical student attitudes toward applying to ophthalmology residency $(n=32)$. Plotted as mean response with $95 \%$ confidence intervals. ${ }^{~ I n d i c a t e s ~ s t a t i s t i c a l l y ~ s i g n i f i c a n t ~}(p<0.05)$ using a one sample $t$-test with a hypothetical mean of 0 . CaRMS, Canadian Residency Matching Service; CI, confidence interval; COVID-19, novel coronavirus 2020.

Respondents mentioned that the shift to virtual interviews could help make the process more equitable due to challenges related to travel and finances. Virtual interviews were noted to potentially allow a student to attend more interviews. There were concerns regarding meeting residents and staff at other programs:

"I worry that since I cannot do out of province electives, I won't get the opportunity to meet with residents and programs directors from various institutions and I won't know what the other programs are like."-Participant no. 2

This was noted by respondents to result in uncertainty when ranking programs. Some respondents preferred the shift to a more online focused education as they said it allowed them more time to study, focus on extra-curricular activities, and complete ophthalmology-related research activities.

\section{Discussion}

This survey demonstrates that Canadian medical students have concerns about how elective changes and lack of inperson CaRMS interviews impact their application to ophthalmology residency. Reconsideration of specialty choice as a result of the COVID-19 pandemic is in keeping with a survey of 627 Canadian medical clerks who found 22\% reconsidered their residency choice. ${ }^{17}$ This survey also found high levels of stress among clerks who was in part due to decreased opportunities to be productive in anticipation of the CaRMS match. ${ }^{17}$ Productivity for medical students is typically defined by involvement in research, volunteer activities, and exposure to clinical experiences. ${ }^{17}$ Students felt lack of out-of-province electives significantly reduced their likelihood of applying and successfully matching to ophthalmology residency. This is similar to other analogous surveys following COVID-19 of U.S. medical students interested in neurosurgery. ${ }^{11}$ Students interested in neurosurgery were more likely to take a year off from medical school in the U.S. sample. ${ }^{11}$ Recent work describing the organization of a webinar focused on mentorship of students interested in ophthalmology highlighted that many existing disparities in the U.S. match, such as lack of research and clinical opportunities for students without an ophthalmology program at their home school, are exacerbated by COVID-19. ${ }^{18}$ Most medical schools in Canada have an ophthalmology residency program, albeit some larger than others that students could be exposed to.

While the shift to online interviews did not affect the likelihood of applying, students also felt a significant decrease in their chances of successfully matching, particularly at programs other than their home institution. Previous work has suggested geographic limitations can play a role in matching to competitive CaRMS specialities. ${ }^{5}$ Our findings are in keeping with an analogous survey of U.S. otolaryngology applicants which found respondents felt less confident about matching, concerns about being fairly assessed in virtual interviews, and inability to gather sufficient information to inform their own rank list. ${ }^{12} \mathrm{~A}$ survey of recently matched urology residents on the interview process in the era of COVID-19 revealed that many thought the faculty interviews could be replicated virtually ( $81 \%)$, but the informal interactions with residents were the most important part (64\%), and this aspect could not be replicated online 
$(81 \%) .{ }^{13}$ This survey of recently matched applicants also emphasized the high value of small virtual encounters with residents. ${ }^{13} \mathrm{~A}$ commentary on the CaRMS match for urology highlights a primary difference between the Canadian and U.S. residency match in that the lack of objective measures, such as USMLE scores, to assess applicants can result in an even greater emphasis on observed clinical performance during rotations. ${ }^{19}$ They argue that lack of travel is even more significant for the CaRMS match compared with the U.S. match as clinical performance is more difficult to assess. ${ }^{19}$

Previous studies have shown value of social media in ophthalmology, focusing mainly on its use in disseminating research and providing general exposure to the field. ${ }^{20,21}$ However in our study, other initiatives were consistently ranked as more valued than programs having a social media presence. A potential explanation is that many applicants will apply to all or the majority of Canadian programs by default and already have an established interest in ophthalmology. Therefore, our study suggests that a social media presence should not be as high of a priority for programs compared with the other more highly valued initiatives including one-on-one video calls. However, one-on-one video calls require a significant time commitment from both program directors and residents, so scheduled "town hall"-type meetings could be a practical intermediate. In Canada, the Association of Faculties of Medicine of Canada (AFMC) created a "National Web Calendar of Events" that has centralized all of these events in one place. ${ }^{22}$ While these events are targeted toward final year medical students, many can be attended by lower year medical students which may help these students explore the specialty of ophthalmology earlier in their training.

A large survey of over 1,000 U.S. medical students found that many felt the COVID-19 pandemic would affect their eventual choice of specialty, citing an inability to explore different fields. ${ }^{23}$ This is particularly important in ophthalmology where exposure in undergraduate medical education is already limited. An international cross-sectional survey reported that many ophthalmology residents felt there would be moderate long-term impacts on their training due to persistent reductions in clinic and surgical volumes. ${ }^{24}$ Lack of shadowing during preclinical years and opportunities for ophthalmology rotations in clinical years may have an impact on future CaRMS cycles beyond the 2021 cycle where students have likely already developed their interest prior to the pandemic. Recent work has described "virtual electives" in ophthalmology in which a student could participate in patient care, ask questions, and have their clinical knowledge probed by a preceptor through various video conferencing applications. ${ }^{25}$ Virtual electives were not discussed in our survey as they have largely emerged following the COVID-19 pandemic, but based on our findings, they are an attractive option. The main limitation is that ophthalmology relies heavily on an in-person physical examination with a slit lamp but virtual solutions, such as a smart phone camera, have been described. ${ }^{19}$ Virtual electives implemented in otolaryngology demonstrated significant educa- tional benefit and produced an increased interest in the field among students. ${ }^{26}$ Additionally, virtual electives can also address the preexisting challenges for students who lack elective availability at their local institution and so may have value beyond the COVID-19 pandemic. Further research in the setting of outpatient ophthalmology to optimize virtual electives would be beneficial.

\section{Limitations and Strengths}

Limitations of this study include our total sample size and lack of a psychometrically validated survey tool. Given social distancing regulations and the time-sensitive nature of this topic, recruitment could only be done by e-mail and the survey could only be open for a limited time which may have limited reach. Validation of our survey tool was in keeping or even more rigorous than analogous time sensitive surveys done in other cohorts. Responses were self-reported which may be subject to bias, as those with more extreme views being more represented or those who lost interest in ophthalmology during the pandemic may not have participated. A strength of this survey is that it recruited participants from a wide variety of medical schools across Canada. We have highlighted the many ways that the CaRMS match is different than the U.S. match and thus warrants independent investigation. Additionally, the response rate relative to total medical student population in our study was much higher relative to analogous U.S. studies in other specialties. Another strength was the optional narrative responses for many questions which allowed students to organically bring ideas and perspectives that were not explicitly asked. Our study revealed several positive outcomes felt by respondents, these ideas have not been widely discussed in other surveys.

\section{Recommendations and Conclusion}

Based on our findings, we propose the following recommendations to Canadian ophthalmology residency programs:

- Organization of accessible opportunities for students to chat directly with program directors and residents through video conference.

- Online information sessions and tours of facilities.

- Communication around electives expectations recognizing the challenges many applicants faced in securing electives at their home institution.

Many similar recommendations have been proposed based on surveys conducted in other small surgical programs such as neurosurgery. ${ }^{27}$ Many prominent ophthalmology educators also highlight the value that online mentorship can have on easing many of the challenges that medical students interested in ophthalmology face during the COVID-19 pandemic. ${ }^{18,28}$ It should be noted that some respondents highlighted the potentially positive outcomes from the COVID-19 pandemic, such as easing the financial barriers for applicants, and these aspects should be leveraged as standards for virtual interviews and online medical education are developed moving forward. Additionally, virtual 
information sessions may help medical students of all levels explore the specialty of ophthalmology, as these opportunities were limited even before the pandemic. Increased acceptance of virtual interactions for networking and research may also help provide equity to those who have been traditionally disadvantaged in the CaRMS match due to their geographic location. Our study suggests the recommendations outlined here would help applicants make an informed decision about their program choice and feel accurately evaluated in the CaRMS match. Moving forward, educators should consider which of these changes should be continued even once the pandemic is over. Despite these challenges, it is encouraging that there remains a sustained interest in ophthalmology among medical students.

Conflict of Interest

None declared.

\section{References}

12020 CaRMS Forum. Accessed August 9, 2020 at: https://www. carms.ca/pdfs/2020-carms-forum.pdf

2 Association of Faculties of Medicine of Canada. COVID-19. Accessed September 7, 2020 at: https://afmc.ca/en/priorities/covid19

3 CaRMS. 2021 match cycle updates. Accessed September 7, 2020 at: https://www.carms.ca/2021-cycle-updates/

4 Mah JM, Kherani IN, Hurley B. Trends in Canadian ophthalmology residency match outcomes. Can Med Educ J 2020;11(03):e67-e72

5 Lakoff J, Howse K, Cofie N, Heeneman S, Dalgarno N. Analysis of factors affecting Canadian medical students' success in the residency match. Can Med Educ J 2020;11(03):e43-e55

6 Wong TY, Bandello F. Academic ophthalmology during and after the COVID-19 pandemic. Ophthalmology 2020;127(08):e51-e52

7 Bargoud AR, Thangamathesvaran L, Patel VR, Henseler R, Kass W, Khouri AS. Quantifying the impact of research on matching into ophthalmology residency. J Acad Ophthalmol 2018;10(01): e133-e139

8 Grubbs JR Jr., Mian SI. Advising students interested in ophthalmology: a summary of the evidence. Ophthalmology 2016;123 (07):1406-1410

9 Venincasa MJ, Cai LZ, Gedde SJ, Uhler T, Sridhar J. Current applicant perceptions of the ophthalmology residency match. JAMA Ophthalmol 2020;138(05):460-466

10 Loh AR, Joseph D, Keenan JD, Lietman TM, Naseri A. Predictors of matching in an ophthalmology residency program. Ophthalmology 2013;120(04):865-870

11 Guadix SW, Winston GM, Chae JK, et al. Medical student concerns relating to neurosurgery education during COVID-19. World Neurosurg 2020;139:e836-e847

12 Izreig S, Torabi SJ, Kasle DA, Rahmati RW, Manes RP. Otolaryngology match 2020-21: survey of prospective applicants in the setting of COVID-19. Ann Otol Rhinol Laryngol 2020 (online ahead of print). Doi: 10.1177/0003489420952470
13 Kenigsberg AP, Khouri RK Jr., Kuprasertkul A, Wong D, Ganesan V, Lemack GE. Urology residency applications in the COVID-19 era. Urology 2020;143:55-61

14 Kapila V, Corthals S, Langhendries L, Kapila AK, Everaert K. The importance of medical student perspectives on the impact of COVID-19. Br J Surg 2020;107(10):e372-e373

15 Kelley K, Clark B, Brown V, Sitzia J. Good practice in the conduct and reporting of survey research. Int J Qual Health Care 2003;15 (03):261-266

16 Standards and guidelines for: response rate. Accessed December 31, 2020 at: www.tpsgc-pwgsc.gc.ca/rop-por/rapports-reports/ comitephone-panelphone/page-06-eng.html

17 Abbas M, Dhane M, Beniey M, et al. Repercussions of the COVID19 pandemic on the well-being and training of medical clerks: a pan-Canadian survey. BMC Med Educ 2020;20(385):. Doi: 10.21203/rs.3.rs-50193/v1

18 Ahmed H, Law JC, Felsted D, et al. Matching ophthalmology amidst coronavirus disease 2019 (COVID-19): lessons that went viral. J Acad Ophthalmol 2020;12(02):e200-e204. Doi: 10.1055/s-00401716412

19 Mann U, Nayak JG. The potential impact of COVID-19 on the Canadian Resident Matching Service: unique future challenges faced by urology residency programs and applicants. Can Urol Assoc J 2020;14(05):E167-E168

20 Schmuter G, Tooley AA, Chen RWS, Law JC. Social media in ophthalmology: the educational and professional potential for medical students. J Acad Ophthalmol 2020;12(01):e41-e45

21 Micieli JA, Tsui E. Ophthalmology on social networking sites: an observational study of Facebook, Twitter, and LinkedIn. Clin Ophthalmol 2015;9:285-290. Doi: 10.2147/OPTH.S79032

22 Association of Faculties of Medicine of Canada. 2021 R-1 match resources. Accessed November 19, 2020 at: https://afmc.ca/en/learners/r1-match

23 Byrnes YM, Civantos AM, Go BC, McWilliams TL, Rajasekaran K. Effect of the COVID-19 pandemic on medical student career perceptions: a national survey study. Med Educ Online 2020;25 (01):1798088

24 Ferrara M, Romano V, Steel DH, et al;OphthaTraining Group. Reshaping ophthalmology training after COVID-19 pandemic. Eye (Lond) 2020;34(11):2089-2097

25 Tsui E, Wells MB, Mohamed M, Felix CM, Giaconi JA. Virtual ophthalmology rotations-a real possibility during the COVID19 pandemic and beyond. J Acad Ophthalmol 2020;12(02): e195-e199

26 Steehler AJ, Pettitt-Schieber B, Studer MB, Mahendran G, Pettitt BJ, Henriquez $\mathrm{OA}$. Implementation and evaluation of a virtual elective in otolaryngology in the time of COVID-19. Otolaryngol Head Neck Surg 2020 (e-pub ahead of print). Doi: 10.1177/0194599820951150

27 Garcia RM, Reynolds RA, Weiss HK, et al. Letter: preliminary national survey results evaluating the impact of COVID-19 pandemic on medical students pursuing careers in neurosurgery. Neurosurgery 2020;87(02):E258-E259

28 Duong AT, Van Tassel SH, Alzaga Fernandez AG, et al. Medical education and path to residency in ophthalmology in the COVID19 era: perspective from medical student educators. Ophthalmology 2020;127(11):e95-e98 